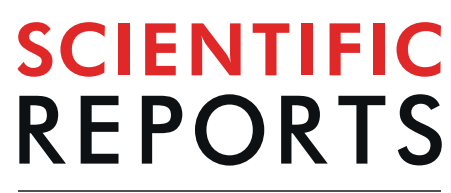

natureresearch

Check for updates

\title{
Pore structure and its impact on susceptibility to coal spontaneous combustion based on multiscale and multifractal analysis
}

\begin{abstract}
Zhang Yu ${ }^{1,2}$, Zhang Xueqing ${ }^{1}$, Yang Wen ${ }^{1,3}$, Xin Haihui ${ }^{4}$, Hu Sherong ${ }^{1 凶}$ \& Song $\mathrm{Yu}^{2 \bowtie}$
The relationship between the properties of coal and its tendency to spontaneous combustion is critical for the environment, safety concerns, and economy. In this study, to eliminate the complex influence of moisture; the samples having similar moisture content were selected from Shanxi and Henan provinces. The chemical properties, physical properties, and tendency of coal samples to spontaneous combustion were characterized based on the conventional analysis, mercury intrusion porosimetry, fractal dimensions, and crossing point temperature (CPT). The results confirmed that the coal rank, volatile matter, oxygen contents, and fixed carbon content had a good linear relationship with the CPT. The relationship between the ash content and CPT presented a "U-shaped" non-linear correlation. For the pore size distribution, the total pore volume also possessed a linear positive correlation with the CPT. The fractal curves could be distinctly divided into two stages: low-pressure ( $<20 \mathrm{MPa}$ ) and highpressure $(>20 \mathrm{MPa})$, from which the fractal dimensions were obtained using the Sponge and Sierpinski models. The relationship between the fractal dimensions $\left(D_{s 1}, D_{s 2}\right.$ and $\left.D_{g 1}\right)$ and CPT could be divided into two distinct stages: a decrease in the CPT with increasing fractal dimensions (2.6-2.85), and then an in increase in the CPT. CPT decreased with increasing parameters of $D_{1}, D_{2}, H$, and $D_{10}$, and it gradually increased with increasing $D_{-10^{-}} D_{10}, D_{-10^{-}} D_{0}$, and $D_{0}-D_{10}$. The above characteristics are important to comprehensively and systematically reveal the mechanism of spontaneous combustion.
\end{abstract}

Low temperature (i.e., $<200^{\circ} \mathrm{C}$ ) chemical and/or physical processes in fossil fuels result in the accumulation of heat, which thereby leads to spontaneous combustion ${ }^{1,2}$. Numerous problems are caused by spontaneous combustion of coal, such as environment issues (emission of greenhouse gases, toxic and harmful gases, and trace elements) ${ }^{3-6}$, safety concerns (dust and gas explosions, unstable overlying rocks, large cracks, and subsidence) ${ }^{7-9}$, and economical loss (destruction of apparatus and equipment, and loss of coal resources) ${ }^{3,10}$. Therefore, it is important to evaluate the degree of proneness for the prevention of the spontaneous combustion of coal.

The crossing point temperature (CPT) is an index to evaluate the tendency of coal to spontaneous combustion $^{11-13}$. Coal properties, oxygen, and ignition are three main factors for predicting the spontaneous combustion tendencies of different coals in chemical and/or physical processes ${ }^{14}$. Numerous studies have been reported on the intrinsic properties of coal and its spontaneous combustion tendency ${ }^{15-20}$. Qi et al. ${ }^{21}$ analyzed the relationship between the CPT and moisture, coal rank, and sulfur content. Nimaje and Tripathy ${ }^{22}$ demonstrated that the parameters of the ultimate analysis had a significant correlation with the Olpinski index, and this relationship could be used as a reliable index to assess the susceptibility of Indian coals to spontaneous combustion. Chandra and Prasad ${ }^{23}$ found that low coal horizons (less than 36\% V.M.; Romax. 0.90-1.25\%) were least susceptible to spontaneous combustion. Pattanaik et al. ${ }^{24}$ demonstrated that the intrinsic properties of coal (stratigraphy or coal rank, volatile matter, and petrography) had a good relationship with the susceptibility indices of spontaneous combustion (CPT, and differential thermal analysis/DTA). Nimaje et al. ${ }^{13}$ analyzed the statistical relationship

${ }^{1}$ College of Geoscience and Surveying, China University of Mining \& Technology, Beijing, 100083, China. ${ }^{2}$ Key Laboratory of Coalbed Methane Resource \& Reservoir Formation Process, Ministry of Education, China University of Mining \& Technology, Xuzhou, 221116, China. ${ }^{3}$ School of Earth Science and Engineering, Hebei University of Engineering, Handan, 056038, China. ${ }^{4}$ School of Safety Engineering, China University of Mining \& Technology, Xuzhou, 221116, China. ${ }^{凶}$ e-mail: hsr8516@126.com; songyu10094488@126.com 
between the CPT and the proximate analysis parameters, and found that mixture surface regression (MSR) model was more effective in predicting spontaneous heating liability of coal.

The influence of water on the self-heat process is complex ${ }^{25}$. Moisture can accelerate the oxidation, and enhance the thermal response by hindering the formation of stabilized radicals ${ }^{25}$. However, the liability of spontaneous combustion of air-dried coal samples increases in the moisture content ${ }^{18}$. Zhang et al. ${ }^{26}$ and Qi et al. ${ }^{21}$ found that moisture may play a role in slowing down spontaneous combustion.

Several pore classification standards have been proposed, which are as follows: 1) Ходот ${ }^{27}$ classified pores $^{-10}$ into micropores ( $<10 \mathrm{~nm}$ in diameter), transitional-pores $(10-100 \mathrm{~nm})$, mesopores $(100-1000 \mathrm{~nm})$, and macropores $(>1000 \mathrm{~nm})$; the International Union of Pure and Applied Chemistry (IUPAC) has classified pores into micropores $(<2 \mathrm{~nm})$, mesopores $(2-50 \mathrm{~nm})$ and macropores $(>50 \mathrm{~nm})$; and Yao et al. ${ }^{28}$ classified pores into adsorption-pores $(<100 \mathrm{~nm})$ and seepage-pores $(100 \mathrm{~nm})$.

Pore structures play an important role in the combustion and oxidation of coal ${ }^{1,29-31}$. Pores provide a good pathway to the transport oxygen to coal surface at low temperature, which increases consumption of oxygen ${ }^{32,33}$. Small space or volume can improve the material performance ${ }^{34}$. Karsner and Perlmutter ${ }^{35}$ found that coal with a large pore volume had high oxidation rate. Parsa et al. ${ }^{1}$ evaluated the effect of densification on brown coal on its physical properties and spontaneous combustion propensity. The results exhibited that the decrease in micropore volume led to a decrease in the contact between oxygen and the coal surface, thereby the increasing the critical ignition temperature. Air can more easily contact the macropore structures of coal (the decreased in the reactivity is initially rapid) than the micropore structures ${ }^{30}$. Mainly investigations on coal structural characteristics such as the pore volume, surface area, pore size distribution, and heterogeneity are conducted to reveal the spontaneous mechanism.

Pore structure characteristics have been widely applied in Coalbed Methane (CBM) exploration and exploitation $^{36-39}$, shale gas reservoirs, and predicting favorable zones ${ }^{40-42}$. Mono fractal approaches have been extensively used in investigating the fractal characteristics of pore structures ${ }^{28,43,44}$. However, a single-scale (mono-fractal) analysis or a single fractal dimension cannot explain the differences in the pore size intervals and types of erratic variation or local variation that occur in the inner distribution of pore sizes ${ }^{45}$. Therefore, a multi-scale fractal model is used for different coal ranks, and tectonically deformed coals with the same coal rank ${ }^{37,46}$. Besides, multifractal methods are also used for non-homogeneous porous media to reveal the heterogeneous pore characteristic ${ }^{47-51}$. Li et al. ${ }^{51}$ and Song et al. ${ }^{45}$ conducted a the multifractal analysis to investigate the variability and heterogeneity of tectonically deformed coals by high-pressure mercury intrusion experiment. Therefore, multifractal analysis is an efficient method to investigate pore size distribution. Nevertheless, focus on the relationship between the spontaneous combustion characteristics and heterogeneity in different coal ranks is lacking.

The objective of this study was to develop an understanding of the relationship between the properties of coal and its tendency to spontaneous combustion. The experiments were conducted by conventional analysis, mercury intrusion porosimetry, and CPT. To eliminate the complex influence of moisture in the process of spontaneous combustion, coal samples having similar moisture contents were selected. This was done to reveal the coal intrinsic properties (coal rank, volatile matter, oxygen contents, fixed carbon contents, and sulfur content), pore characteristics (pore volume, surface area, pore size distribution, and heterogeneity), and tendency of coal to spontaneous combustion. Both multiscale and multifractal methods were used to systematically investigate the heterogeneity and susceptibility to coal spontaneous combustion. Such research is important to comprehensively and systematically reveal the mechanism of spontaneous combustion.

\section{Experiments and Modelling}

Geological setting. A total of seven coal samples were collected from Shanxi and Henan provinces (Fig. 1), which contain the major coalfields in China. Six samples were collected from three coalfields in Shanxi, including Ningwu, Xishan, and Qinshui, and one sample was collected from Henan coalfield (Pingdingshan). The main coal-bearing stratum of these coalfields belongs to the Carboniferous-Permian system. Samples were directly collected from coal colliery following the Chinese Standard Method GB/T 19222-2003 and were carefully packed and taken to the laboratory for experiments.

Samples and experiments. Seven coals were collected from Ningwu, Dongqu, Guandi, Longle, Fenghuangshan, and Pingdingshan collieries in Shanxi and Henan provinces. The coal samples were crushed to -200 meshes $(\sim 0.074 \mathrm{~mm})$ and vacuum-dried. The ultimate analysis and proximate analysis were conducted in accordance with the Chinese national testing standards (GB/T 212-2008 and GB/T 31391-2015). Vitrinite random reflectance $(\% \mathrm{Rr})$ were measured for all the coal samples on the same polished sections using a Leitz MPV-3 photometer microscope, following conventional methods in accordance with the Chinese national standard (GB/T 6948-1998).

The CPT measurements were conducted at the China University of Mining and Technology. The details of the experiment are reported in our previous work ${ }^{26}$. The samples (mass $50 \mathrm{~g}$ with a particle size of $\sim 0.18-0.43 \mathrm{~mm}$ ) were exposed to a dry air flow of $50 \mathrm{~mL} / \mathrm{min}$ within the reactor with a temperature ramp rate of $1^{\circ} \mathrm{C} / \mathrm{min}$ with coal and oven temperatures being recorded. When the coal temperature is equal to the oven temperature, the sample is at the $\mathrm{CPT}^{21,52}$.

The coal samples were prepared by a vacuum drying for $12 \mathrm{~h}$ at $70-80^{\circ} \mathrm{C}$. High pressure mercury injection (HPMI) experiment was performed for the samples using an Auto Pore IV 9510 HPMI instrument at the China University of Mining and Technology. The mercury injection pressure ranged from 0.90 to $4.0 \times 10^{4} \mathrm{PSIA}$. The pore diameters obtained were from 5.35 to $2.28 \times 10^{5} \mathrm{~nm}$.

Fractal dimensions. Sierpinski model. Based on the Sierpinski model ${ }^{53}$, the fractal dimensions $\left(D_{s}\right)$ can be calculated by the following equation: 


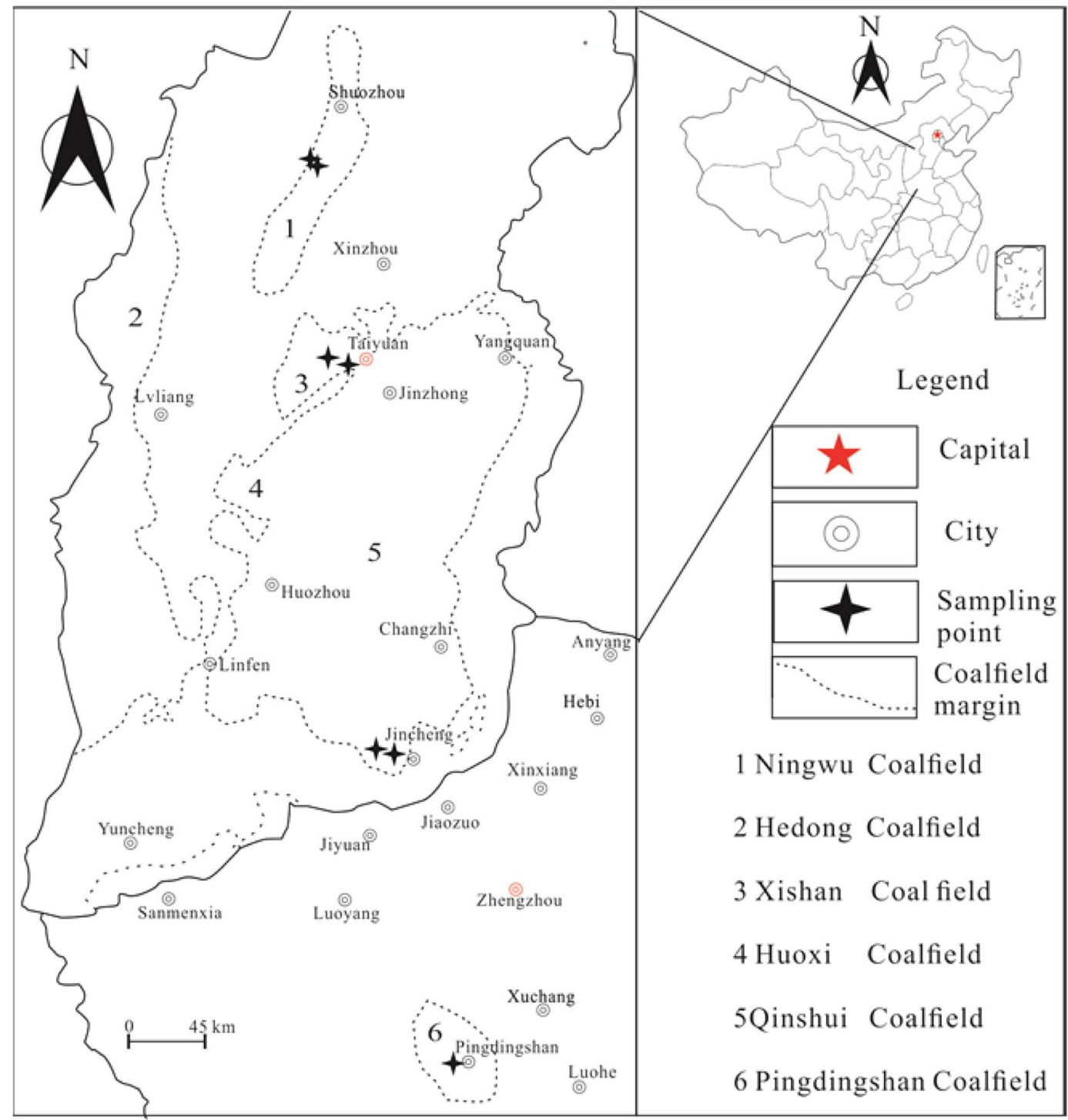

Figure 1. Positions of the research areas and the distribution of the sampling points.

$$
\ln (V)=(3-D s) \ln (P-P t)+\ln \alpha
$$

where $V$ is the mercury injection amount at $P$ in $\mathrm{mL}, D$ is the volume fractal dimension, $P_{\mathrm{t}}$ is the mercury inlet pressure in $\mathrm{MPa}$, and $\alpha$ is a constant. If the slope of the $\ln \left(P-P_{t}\right) v s$. $\ln (V)$ curve is $K$, then $D_{s}$ is $3-K$.

Sponge model. Based on the Menegr model ${ }^{53}$, the fractal dimensions (Dg) can be calculated by the following equation:

$$
D g=\ln [d V P(r) / d P(r)]-\ln \alpha / \ln P(r)+4,
$$

where $V_{P(r)}$ is the cumulative injection volume at a given pressure $P(r)$ and $\alpha$ is a constant. Therefore, the pore fractal dimension, $D_{g}$, can be obtained by: $D_{g}=4+A$, where $\mathrm{A}$ is the slope in Eq. (2).

Multifractals. Multifractal analysis is used to measure the statistic Hg pore size distributions. The pore diameter interval $(I)$ ranging from $0.006 \mathrm{~nm}$ to initial diameter (responding to the least pressure) was selected to generate a box $\left(N(\varepsilon)=2^{k}, \varepsilon=\mathrm{L} \times 2^{k}\right)$ by dyadic partitions in $\mathrm{k}$ stages $(k=1,2,3, \ldots)$, where $L$ is the length of the support $^{45,48,50,51}$. The probability, $P_{\mathrm{j}}(\varepsilon)$, of the pore size can be calculated as ${ }^{48}$,

$$
P_{j}(\varepsilon)=\frac{N_{j}(\varepsilon)}{N_{t}},
$$

where, $N_{j}(\varepsilon)$ is the volume of a box $(j=1,2,3 \ldots)$, and $N_{t}$ is the total volume of the system.

The probability for each box of size $\varepsilon$ unit can be calculated $\mathrm{as}^{48}$, 


$$
P(\varepsilon)=\varepsilon^{-a j},
$$

where $\alpha_{j}$ is the coarse Hölder or singularity exponent for the boxes, which theoretically represents how the singularities of a system tend to infinity in the limit $\varepsilon \rightarrow 0$.

The $\alpha$ exponent, $N_{\alpha}$, was used to evaluate the number of boxes, as follows:

$$
N \alpha(\varepsilon) \alpha^{-f(\alpha)},
$$

where the set of $f(\alpha)$ values represents the spectrum of fractal dimensions that characterize the abundance of the set of points with singularity $\alpha . f(\alpha)$ can be calculated s $^{48}$ :

$$
a(q) \propto \frac{\sum_{j=1}^{N(\varepsilon)} u_{j}(q, \varepsilon) \log \left\{p_{j}(\varepsilon)\right\}}{\log (\varepsilon)}
$$

and

$$
f\{\alpha(q)\} \propto \frac{\sum_{j=1}^{N(\varepsilon)} u_{j}(q, \varepsilon) \log \left\{u_{j}(q, \varepsilon)\right\}}{\log (\varepsilon)},
$$

where $\mu_{j}(q, \varepsilon)$ and $P_{j}(\varepsilon)$ are the normalized measures, defined $\operatorname{as}^{48}$ :

$$
\begin{aligned}
\mu_{j}(q, \varepsilon) & =\frac{p_{j}^{q}(\varepsilon)}{\sum_{j=1}^{N(\varepsilon)} \sum_{j}^{p}(\varepsilon)} \\
\chi(q, \varepsilon) & =\sum_{j=1}^{N(\varepsilon)} p_{j}^{q}(\varepsilon),
\end{aligned}
$$

where $\chi(q, \varepsilon)$ can be calculated as:

$$
\chi(q, \varepsilon) \propto \varepsilon^{-\tau(q)},
$$

where $\tau(q), q$ th mass exponent, can be defined as $^{48}$,

$$
\tau(q)=\lim _{\varepsilon \rightarrow 0} \frac{\log \mu(q, \varepsilon)}{\log \left(\frac{1}{\varepsilon}\right)}=(1-q) D_{q},
$$

where $D_{q}$ called the generalized fractal dimensions or Rényi dimensions can be calculated as ${ }^{48}$,

$$
\begin{gathered}
D_{q}=\frac{1}{q-1} \lim _{\varepsilon \rightarrow 0} \frac{\log \mu(q, \varepsilon)}{\log \varepsilon}(q \neq 1), \\
\mathrm{D}_{1}=\frac{\sum_{j=1}^{N(\varepsilon)} p_{j} \log p_{j}}{\log (\varepsilon)}(q=1),
\end{gathered}
$$

\section{Results and data analyses}

Conventional characteristics. The vitrinite random reflectance $(\mathrm{Rr}, \%)$ of the coal samples was ranged from $0.58 \%$ to $3.43 \%$, corresponding to medium-rank coal (bituminous coals A, B, C, and D) to high-rank coal (anthracite coals B and C) (ISO 11760, 2005) ${ }^{54}$. Proximate analysis showed that the volatility of the coals varied from $5.95 \%$ to $43.45 \%$. The moisture content was similar in different coal ranks. The fixed carbon ranged from $27.83 \%$ to $80.88 \%$. The total sulfur content changed from $0.42 \%$ to $9.36 \%$. The ash yields also had a wide range, varying from $5.47 \%$ to $49.09 \%$ (Table 1 ).

Macropores structure from HPMI experiments. Pore structure distribution. Table 2 lists the pore parameters obtained from the HPMI. In this study, the classification standards defined by Yao et al. ${ }^{28}$ and the sharpness of the curve were used. The following three ranges are present: $\mathrm{V}_{1}<100 \mathrm{~nm}, 100<V_{2}<1000 \mathrm{~nm}$, and $V_{3}>10000 \mathrm{~nm}$. Samples $\mathrm{S}_{2}$ and $\mathrm{S}_{1}$ have the largest and smallest cumulative pore volume in $V_{1}$, respectively. Cumulative pore volumes $V_{2}$ have a relatively lower distribution than $V_{1}$ or $V_{3}$, and among the samples, sample $\mathrm{S}_{3}$ has the highest distribution. The coal rank ranges from bituminous $\mathrm{A}$ to anthracite $\mathrm{B}$ when the cumulative pore volume, $V_{2}$, is the same $\left(0.002 \mathrm{~cm}^{3} / \mathrm{g}\right)$. Samples $\mathrm{S}_{1}$ and $\mathrm{S}_{3}$ have the smallest and largest cumulative pore volumes $V_{3}$, respectively, which are in response to the total pore volume.

The mercury injection and withdraw curves as well as the pore size distribution are shown in Fig. 1a,b, respectively. For the high-rank coals, the mercury intrusion and extrusion curves (Fig. 2a) display a similar trend (parallel type), indicating the dominance of the parallel plate pores and a good connectivity for gas diffusion. The shape increases in case of mercury intrusion at low pressures reveal a high proportion of $\mathrm{V}_{3}$, and the slightly straight lines reveal a poorly developed $\mathrm{V}_{1}$, which are in agreement with pore volume distribution. For the medium-rank coals, 


\begin{tabular}{|c|c|c|c|c|c|c|c|c|c|c|c|c|}
\hline \multirow[b]{2}{*}{ Samples } & \multirow[b]{2}{*}{ Coal coalfield } & \multirow{2}{*}{$\begin{array}{l}\mathbf{R r} \\
(\%)\end{array}$} & \multirow[b]{2}{*}{ Coal ranks } & \multicolumn{4}{|c|}{ Proximate analysis. \% } & \multicolumn{5}{|c|}{ Ultimate analysis. \% } \\
\hline & & & & $M_{d}$ & $A_{d}$ & $V_{d}$ & $\mathrm{FC}_{\mathrm{d}}$ & $\mathrm{S}_{\mathrm{t} . \mathrm{d}}$ & $\mathbf{O}_{\text {daf }}$ & $\mathrm{C}_{\text {daf }}$ & $\mathrm{H}_{\text {daf }}$ & $\mathrm{N}_{\mathrm{daf}}$ \\
\hline $\mathrm{S}_{1}$ & Ningwu & 0.58 & Bituminous D & 1.02 & 49.09 & 23.07 & 27.83 & 9.36 & 12.36 & 63.23 & 4.95 & 1.08 \\
\hline $\mathrm{S}_{2}$ & & 0.63 & Bituminous C & 1.01 & 5.47 & 43.45 & 51.09 & 2.51 & 8.49 & 81.83 & 5.66 & 1.37 \\
\hline $\mathrm{S}_{3}$ & Pingdingshan & 1.31 & Bituminous B & 1.00 & 38.81 & 18.17 & 43.02 & 0.42 & 3.69 & 80.61 & 4.43 & 1.57 \\
\hline $\mathrm{S}_{4}$ & Dongqu & 1.59 & Bituminous A & 1.01 & 6.47 & 14.82 & 78.79 & 1.78 & 4.76 & 87.88 & 4.13 & 1.32 \\
\hline $\mathrm{S}_{5}$ & Guandi & 2.13 & Anthracite C & 1.01 & 9.40 & 9.71 & 80.88 & 1.79 & 3.36 & 89.73 & 3.69 & 1.23 \\
\hline $\mathrm{S}_{6}$ & Longle & 3.08 & Anthracite B & 1.02 & 26.61 & 7.04 & 66.35 & 4.94 & 3.89 & 86.13 & 2.52 & 0.74 \\
\hline $\mathrm{S}_{7}$ & Fenghuangshan & 3.43 & Anthracite B & 1.01 & 16.03 & 5.95 & \begin{tabular}{|l|l|}
78.02 \\
\end{tabular} & 5.47 & 0.85 & \begin{tabular}{|l|l|}
89.28 \\
\end{tabular} & 2.61 & 0.75 \\
\hline
\end{tabular}

Table 1. Properties of the coal samples having different coal ranks.

\begin{tabular}{|c|c|c|c|c|c|c|}
\hline \multirow[b]{2}{*}{ Samples } & \multicolumn{3}{|c|}{ Cumulative pore volume $\left(\mathrm{cm}^{3} / \mathrm{g}\right)$} & \multirow{2}{*}{$\begin{array}{l}\text { Mean volume } \\
\left(\mathrm{cm}^{3} / \mathrm{g}\right)\end{array}$} & \multirow{2}{*}{$\begin{array}{l}\text { Mean surface } \\
\text { area }\left(\mathrm{m}^{2} / \mathrm{g}\right)\end{array}$} & \multirow{2}{*}{$\begin{array}{l}\text { Total pore } \\
\text { volume }\left(\mathrm{cm}^{3} / \mathrm{g}\right)\end{array}$} \\
\hline & $V_{1}$ & $V_{2}$ & $V_{3}$ & & & \\
\hline $\mathrm{S}_{1}$ & 0.011 & 0.009 & 0.006 & 0.018 & 0.306 & 0.026 \\
\hline $\mathrm{S}_{2}$ & 0.039 & 0.003 & 0.024 & 0.030 & 0.369 & 0.066 \\
\hline $\mathrm{S}_{3}$ & 0.015 & 0.014 & 0.005 & $*$ & $*$ & 0.033 \\
\hline $\mathrm{S}_{4}$ & 0.024 & 0.002 & 0.105 & 0.115 & 0.158 & 0.130 \\
\hline $\mathrm{S}_{5}$ & 0.021 & 0.002 & 0.115 & 0.120 & 0.062 & 0.137 \\
\hline $\mathrm{S}_{6}$ & 0.015 & 0.002 & 0.075 & 0.082 & 0.097 & 0.092 \\
\hline $\mathrm{S}_{7}$ & 0.019 & 0.002 & 0.202 & 0.020 & 0.204 & 0.223 \\
\hline
\end{tabular}

Table 2. Results of the HPMI and pore distribution for different coal ranks. Note: $V_{1}, V_{2}$, and $V_{3}$ are the cumulative pore volumes of $<100 \mathrm{~nm}, 100-10000 \mathrm{~nm}$, and $>10000 \mathrm{~nm}$ respectively, and $V_{t}=V_{1}+V_{2}+V_{3}$. *no data.
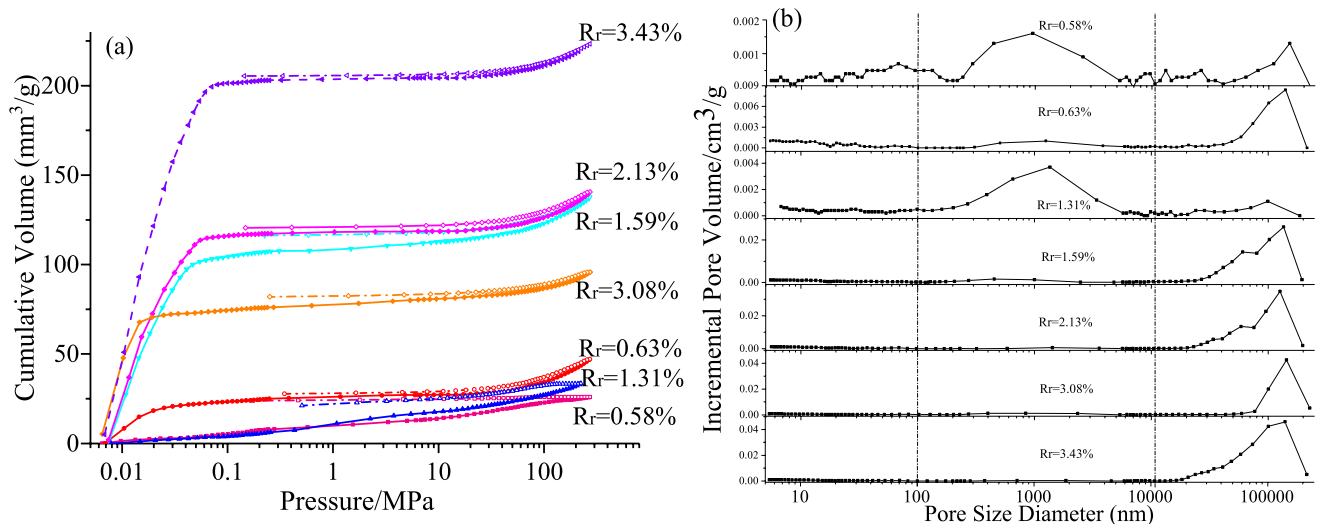

Figure 2. (a) Mercury injection and withdraw curves, and (b) Pore size distribution.

there is larger space between the mercury intrusion and extrusion curves (hysteresis loop) (Fig. 2a) than between those of the high-rank coals (tip-edge type), indicating a larger $\mathrm{V}_{1}$, small $\mathrm{V}_{2}$ and $\mathrm{V}_{3}$, and poorer pore connectivity than that in the high-rank coals. The larger hysteresis loop suggests a significant existence of the semi- closed pores. The pore size distribution curves (Fig. 2b) remarkably change with the increase in the coal rank.

Fractal dimensions by Sierpinski model. The fractal curves based on the Sierpinski model are presented in Fig. 3. Fractal dimension $D_{s 1}$ and $D_{s 2}$ are obtained in low-pressure ( $<20 \mathrm{MPa}$, responding to the see page pores, $>100 \mathrm{~nm}$ ) and high-pressure ( $<20 \mathrm{MPa}$, responding to adsorption pores, $<100 \mathrm{~nm}$ ) stages. The correlation index of $D_{s 1}$ ranges from 0.30 to 0.99 . Medium-rank coals $\mathrm{S}_{1}, \mathrm{~S}_{2}$ and $\mathrm{S}_{3}$ and high-rank coal $\mathrm{S}_{5}$ present a better correlation. However, the correlation index of $D_{s 2}$ had a high correlation index $(0.80-0.995)$. The values of $D_{s 1}$ and $D_{s 2}$ ranges from 2 to 3, indicating their power law relationship with the fractal pore surface. The values of $D_{s 1}$ and $D_{s 2}$ are $2.64-2.98$ (2.90 in average) and 2.80-2.98 (2.88 in average), respectively.

Fractal dimensions by sponge model. The fractal curves are divided into two stages based on the classic geometry model (sponge model) (Fig. 4), and thus two fractal dimensions $\left(D_{g 1}\right.$, low-pressure and $D_{g 2}$, high-pressure) are obtained using the mercury intrusion data. $D_{g 1}$ exhibits a good linear relationship (correlation index $R^{2}$, 

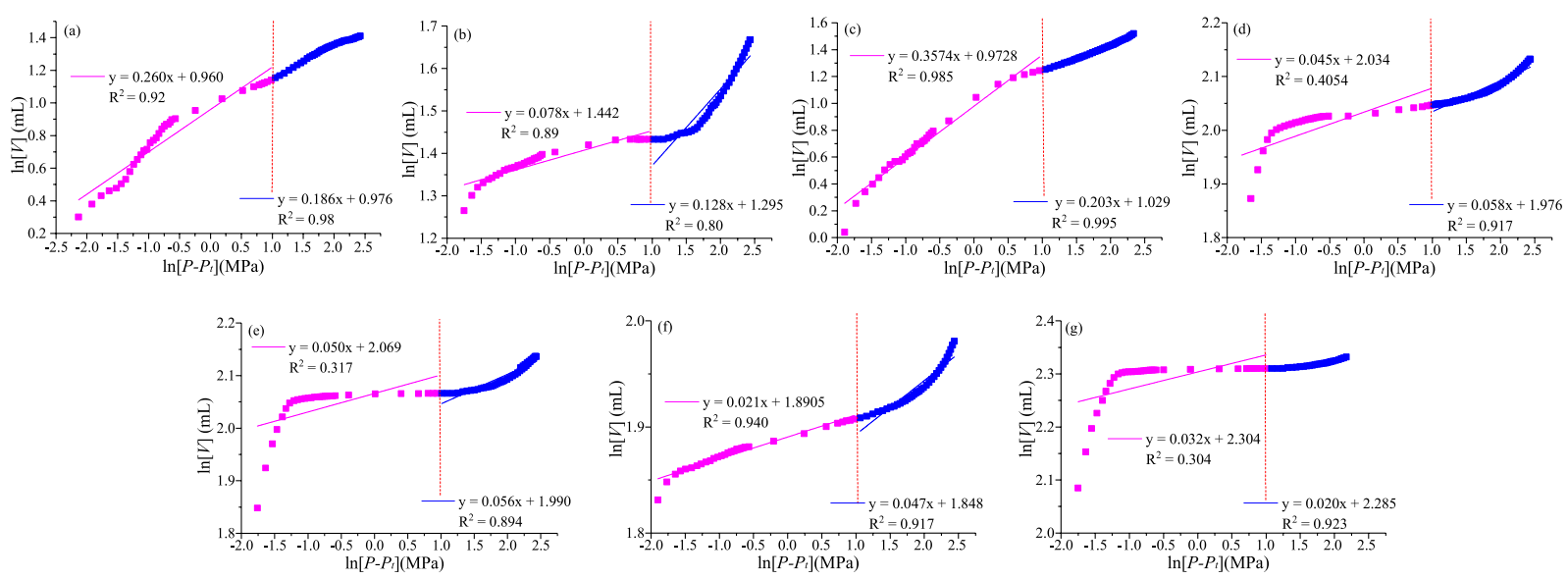

Figure 3. Fractal dimensions $\mathrm{D}_{\mathrm{s} 2}$ and $\mathrm{D}_{\mathrm{s} 1}$ for different coal ranks based on the Sierpinski model.
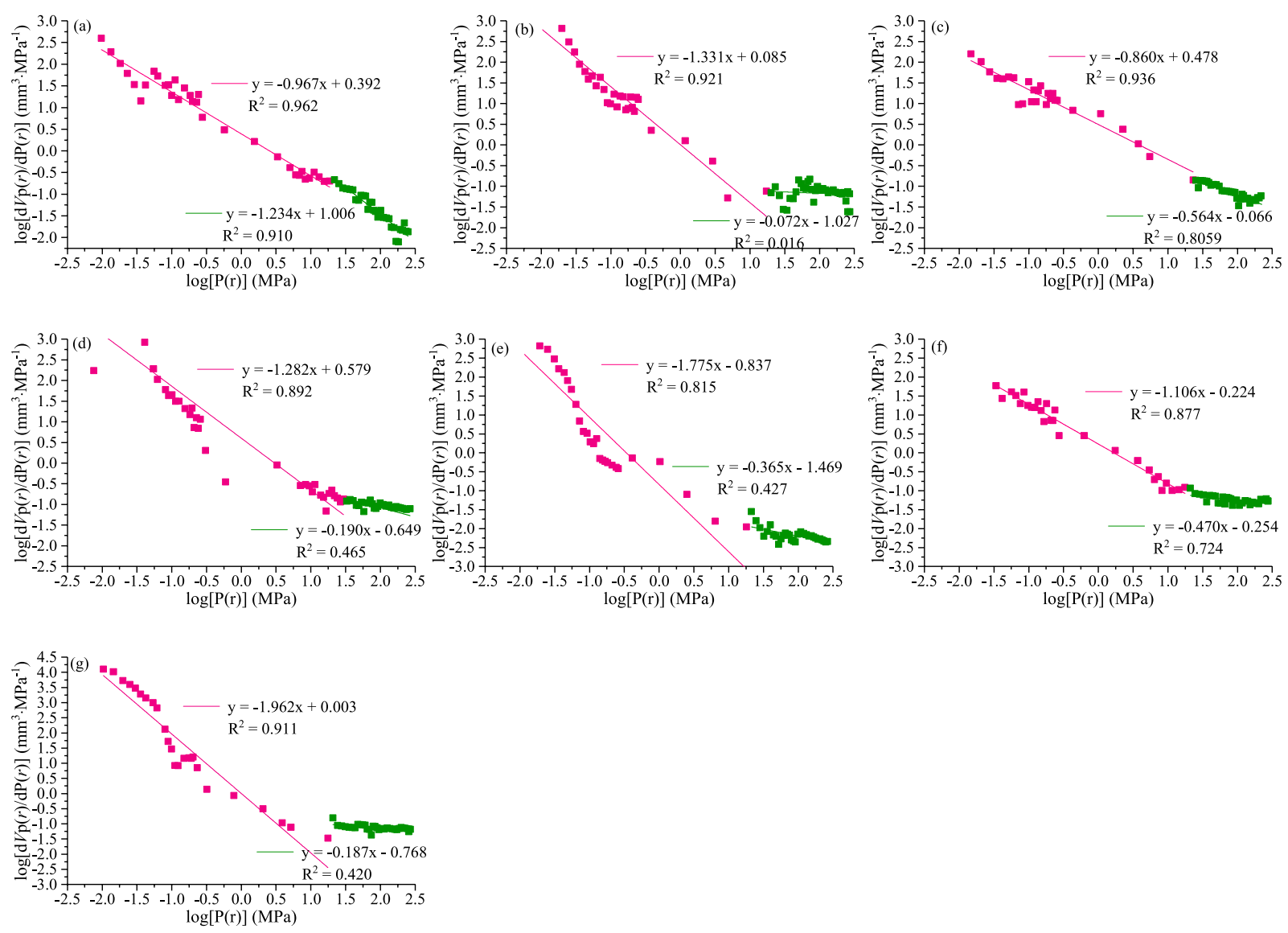

Figure 4. Fractal dimensions Ds 2 and $D s 1$ for different coal ranks based on the sponge model.

$0.82-0.96)$, whereas $D_{g 2}$ has a wide range of $\mathrm{R}^{2}(0.02-0.91)$. The values of $D_{g 1}$ are widely distributed (2.04-3.14, 2.67 in average), indicating significant differences in the discontinuities and roughness of different coal ranks. However, the value of $D_{g 2}$ are 2.77-3.93 (3.56 in average). Most of the values of $D_{g 2}$ are close to 3, indicating that the surface is extremely rough and the pore structure is irregular ${ }^{55}$. The values of $D_{g 1}$ in $\mathrm{S}_{1}$ and $\mathrm{S}_{3}$ are $>3$, and all the values of $D_{g 2}$ are more than 3, except for sample $S_{1}$. For the fractal dimensions of 3 , numerous explanations have been provided in previous research ${ }^{28,44}$.

Multifractal analysis. The spectrum curves of $\log \chi(\mathrm{q}, \varepsilon)$ versus $\log \varepsilon$ show a linear relationship (correlation index $\left.R^{2}, 0.78-1\right)$, indicating a multifractal distribution of the pore sizes ${ }^{56}$ (Fig. 5). The spectrum curves of the generalized dimensions, $D(q)$, versus q present a sigma-shaped curve and follow a monotone decreasing function of $q$ (Fig. 6a). The characteristic parameters of $D(q)$, dimensions $D_{0}, D_{1}$, and $D_{2}$, Hurst exponent $H\left(2 H=D_{2}+1\right)$, 

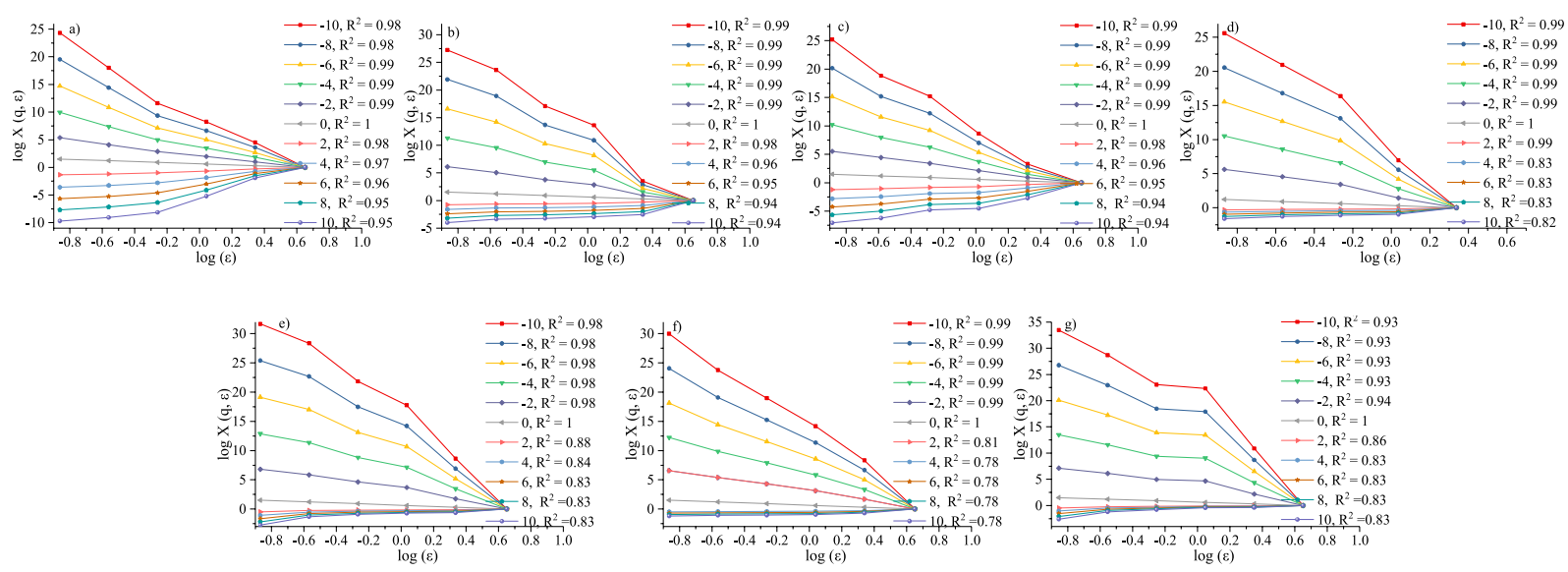

Figure 5. Partition function, $\log \chi(q, \varepsilon)$, box size, and $\log \varepsilon$ for the pore size distributions.
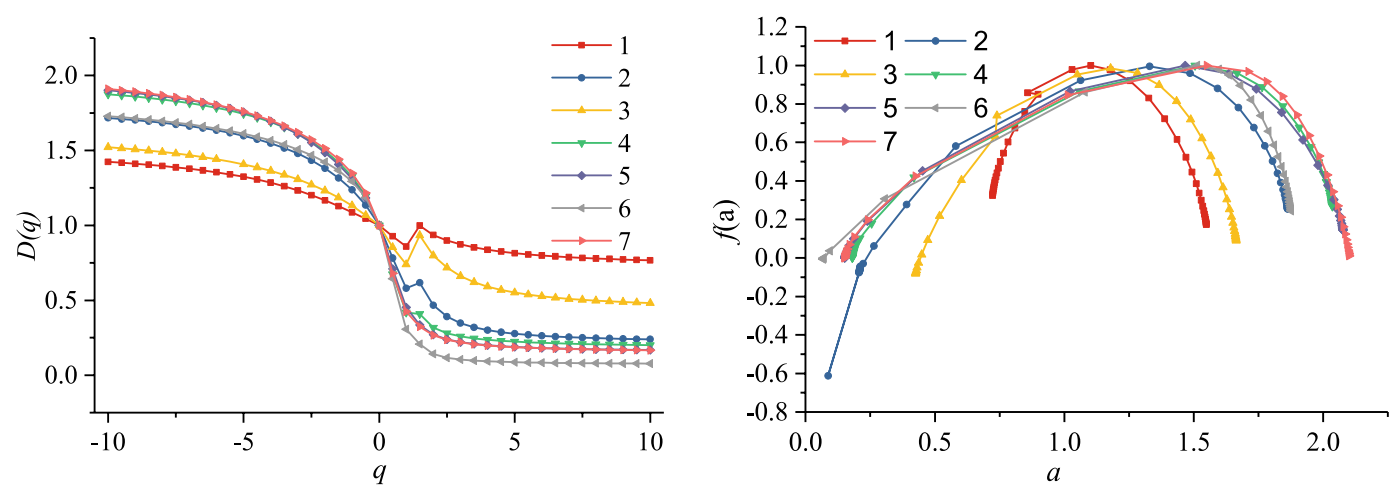

Figure 6. (a) Curve of Dq versus $\mathrm{q}(-10,10)$ of the coal samples and (b) the multifractal singularity spectra of the coal samples.

\begin{tabular}{|l|l|l|l|l|l|l|l|l|l|l|}
\hline Samples & $\mathbf{D}_{\mathbf{0}}$ & $\mathbf{D}_{\mathbf{1}}$ & $\mathbf{D}_{\mathbf{2}}$ & $\mathbf{H}$ & $\mathbf{D}_{\mathbf{1 0}}$ & $\mathbf{D}_{-\mathbf{1 0}}$ & $\mathbf{D}_{-\mathbf{1 0}}-\mathbf{D}_{\mathbf{1 0}}$ & $\mathbf{D}_{\mathbf{0}}-\mathbf{D}_{\mathbf{1 0}}$ & $\mathbf{D}_{-\mathbf{1 0}}-\mathbf{D}_{\mathbf{0}}$ & $\mathbf{a}_{\mathbf{0}}$ \\
\hline $\mathrm{S}_{1}$ & 1 & 0.86 & 0.94 & 0.97 & 0.77 & 1.42 & 0.66 & 0.23 & 0.42 & 1.10 \\
\hline $\mathrm{S}_{2}$ & 1 & 0.58 & 0.47 & 0.73 & 0.24 & 1.72 & 1.48 & 0.76 & 0.72 & 1.33 \\
\hline $\mathrm{S}_{3}$ & 1 & 0.74 & 0.80 & 0.90 & 0.48 & 1.52 & 1.04 & 0.52 & 0.52 & 1.18 \\
\hline $\mathrm{S}_{4}$ & 1 & 0.42 & 0.32 & 0.66 & 0.20 & 1.88 & 1.67 & 0.80 & 0.88 & 1.51 \\
\hline $\mathrm{S}_{5}$ & 1 & 0.45 & 0.27 & 0.64 & 0.17 & 1.90 & 1.73 & 0.83 & 0.90 & 1.47 \\
\hline $\mathrm{S}_{6}$ & 1 & 0.31 & 0.14 & 0.57 & 0.08 & 1.73 & 1.65 & 0.92 & 0.73 & 1.51 \\
\hline $\mathrm{S}_{7}$ & 1 & 0.42 & 0.27 & 0.63 & 0.17 & 1.91 & 1.74 & 0.83 & 0.91 & 1.55 \\
\hline
\end{tabular}

Table 3. Multifractal parameters from the generalized dimension spectrum. $D_{0}$, the capacity dimension; $D_{1}$, the entropy dimension; $\mathrm{D}_{2}$, the correlation dimension; $\mathrm{H}$, Hurst exponent; $\mathrm{D}_{10}$ and $\mathrm{D}_{-10}$ are the generalized dimensions responding to $\mathrm{q}=10$ and $\mathrm{q}=-10$, respectively.

width $D_{-10}-D_{10}$ of $D(q)$ spectrum, right side width $D_{0}-D_{10}$, and left side width $D_{-10}-D_{0}$ (Table 3 ), reflect the inner variability and heterogeneity of the porosity and pore size distribution ${ }^{45,51,57}$. Samples $S_{1}$ and $S_{6}$ have the highest and lowest values of $D_{1}, D_{2}, H$, and $D_{10}$, respectively. The values of $D_{1}$ widely range from 0.31 to 0.86 .

Propensity to spontaneous combustion. Fig. 7 displays the results of the CPT measurements performed on the medium-rank and high-rank coals. The CPT ranges from 146.1 to $182.2^{\circ} \mathrm{C}$. Samples $\mathrm{S}_{7}$ (anthracite B) and $\mathrm{S}_{1}$ (bituminous $\left.\mathrm{D}\right)$ have the highest $\mathrm{CPT}\left(182.2^{\circ} \mathrm{C}\right)$ and the lowest $\mathrm{CPT}\left(146.1^{\circ} \mathrm{C}\right)$, respectively. The $\mathrm{CPT}$ increases with increasing coal rank.

\section{Discussion}

Effect of evolution of coal petrology on spontaneous combustion. Relationship between $\mathrm{Rr}$ and $C P T$ and pore structure. Coal rank has a significant influence on the propensity of coal to spontaneous combustion (Fig. 8a). A good linear relationship $\left(\mathrm{R}^{2}=0.74\right)$ is exhibited between the coal rank and the CPT. The CPT increases with increasing coal rank, which is in agreement with previous studies ${ }^{58-61}$. However, sample $\mathrm{S}_{6}$ exhibits 

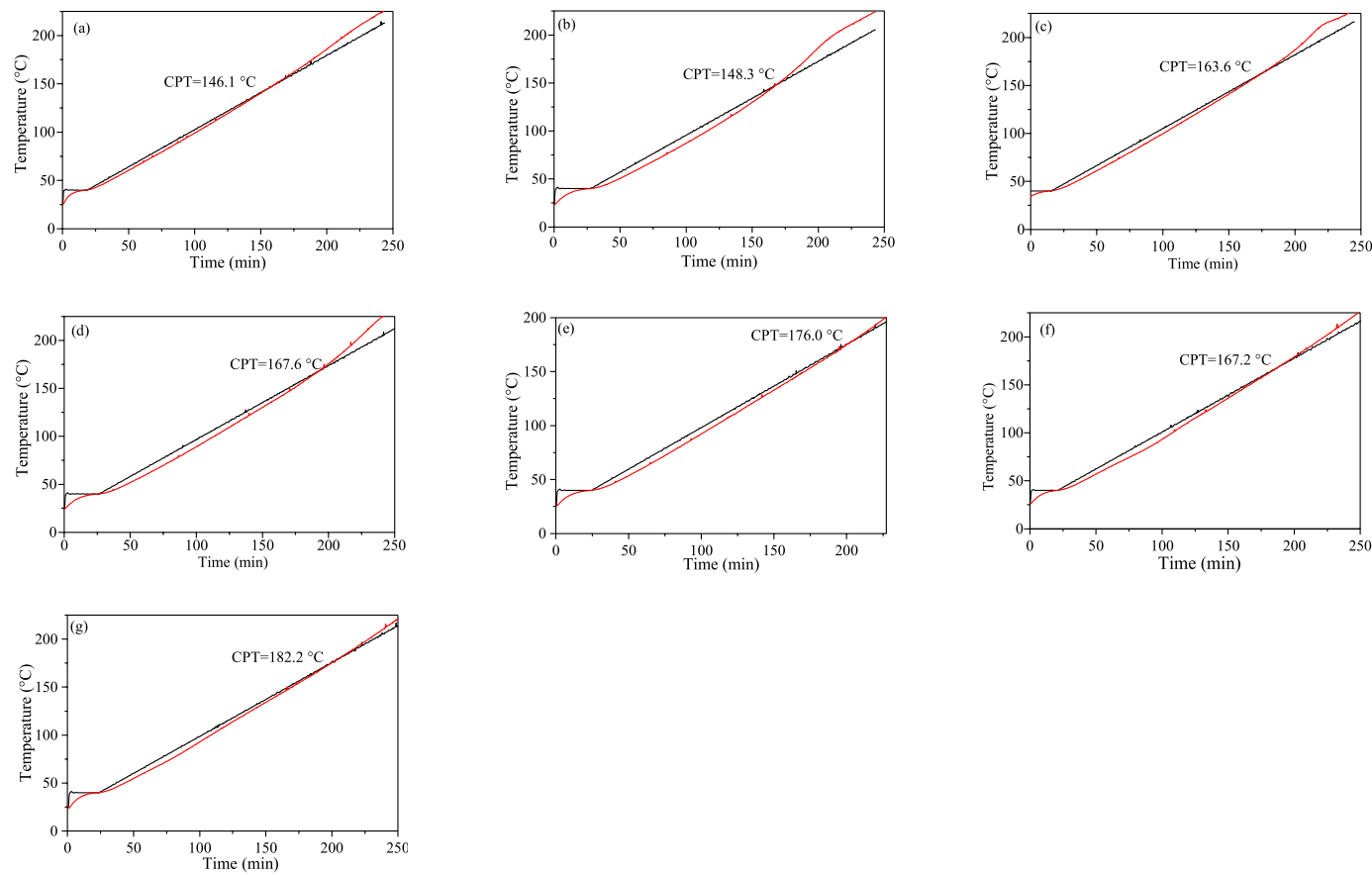

Figure 7. CPT for the different coal ranks.

a deviation compared to the other samples. If this sample data are removed, a better linear $\left(\mathrm{R}^{2}=0.89\right)$ is obtained, as shown in Fig. 8a. For the high-rank sample $\mathrm{S}_{6}$, the low CPT may be attributed to its different chemical (low fixed carbon) and physical (low total pore volume) structure, which is in agreement with previous studies ${ }^{11,12}$. Coal rank is an important index of coalification, influencing the structures of coal pores and fractures. When the vitrinite random reflectance ranges from 0.58 to $2.13 \%$ (Fig. $8 \mathrm{~b}$ ), the mean volume increases with the coal rank, but the mean surface area decreases. When the vitrinite random reflectance ranges from 2.13 to $3.43 \%$ (Fig. $8 \mathrm{c}$ ), the mean volume decreases with coal rank, but the mean surface area increases with coal rank. When the coalification ranges from 0.5 to $2.1 \%$, the aromatic structures including non-protonated aromatic carbons $\left(\mathrm{fa}^{\mathrm{N}}\right)$, nuclear magneton resonance (NMR) aromaticity $\left(f a^{\prime}\right)$, and aromatic carbon ratio ( $f a$ ) increase linearly, whereas the aliphatic structures decreases linearly ${ }^{62}$. Because the volume is mainly affected by the aliphatic parts of the chemical structure, it may be the cause of the displayed mean volume and surface area trends ${ }^{63,64}$.

Relationship between coal composition and CPT. There exists a non-linear relationship between the ash content and the CPT, which shows an inverted 'U-shape' (Fig. 9a). The CPT is a weakly correlated to the total sulfur content (Fig. 9b). The coal components (volatile matter, oxygen contents, and fixed carbon contents) display good relationships with the CPT (Fig. 9c-e), which is in agreement with Zonguldak coals ${ }^{65}$. The CPT decreases with increasing volatile matter, illustrating that low volatile matter is prone to spontaneous combustion. It may be that low volatile matter content can increase the difficulty of ignition and result in an unstable combustion flame ${ }^{66,67}$. The relationship between the oxygen content and the CPT also shows the same trend as the volatile matter, indicating that coals with high oxygen content have a high tendency to chemically bind moisture, thereby rendering the surface, highly susceptible to autogenous heating ${ }^{68}$. The CPT increases linearly with the fixed carbon content, indicating that less fixed carbon content is prone to spontaneous combustion. This is because a small amount of fixed carbon in coal requires a low activation energy to initiate combustion ${ }^{69}$.

Relationship between pore structure and CPT. Distribution of pore structure and CPT. It is necessary to discuss the relationship between the pore size and the CPT. Fig. 10a shows a weak negative linear correlation between the cumulative pore volume $V_{1}$ and the CPT, which shows an inverted " $\mathrm{U}$-shape". Cumulative pore volume $V_{2}$ shows a "U-shape"(Fig. 10b). The relationship between cumulative pore volume $V_{3}$ and the CPT exhibit a linear positive correlation (Fig. 10c). Moreover, the total pore volume also has a good linear positive correlation with the CPT ( $R^{2}$ up to 0.71 ), indicating that pore sizes of more than $10000 \mathrm{~nm}\left(V_{3}\right)$ play a main role in coal spontaneous combustion (Fig. 10d). Specifically, the pore structure is a dominant factor causing the coal spontaneous combustion, particularly in pore sizes more than $10000 \mathrm{~nm}$.

Relationship between coal rank, pore volume, and multifractal parameters. The entropy dimension $\left(D_{1}\right)$ reveals the concentration degree of the porosity distribution ${ }^{48}$. The values of $D_{1}$ are less than or equal to $\mathrm{D}_{0}$. When $D_{1}$ is close to $D_{0}$, the porosity is evenly distributed. Otherwise, most particles are concentrated in a small area and appear as a high peak on the graph ${ }^{70}$. Among of all sample $S_{1}$ has the highest homogeneous pore size distribution. $D_{1}$ decreases with increasing coal rank (Fig. 11a), indicating an increase in the heterogeneity. However, the fitting results do not a high correlation. 

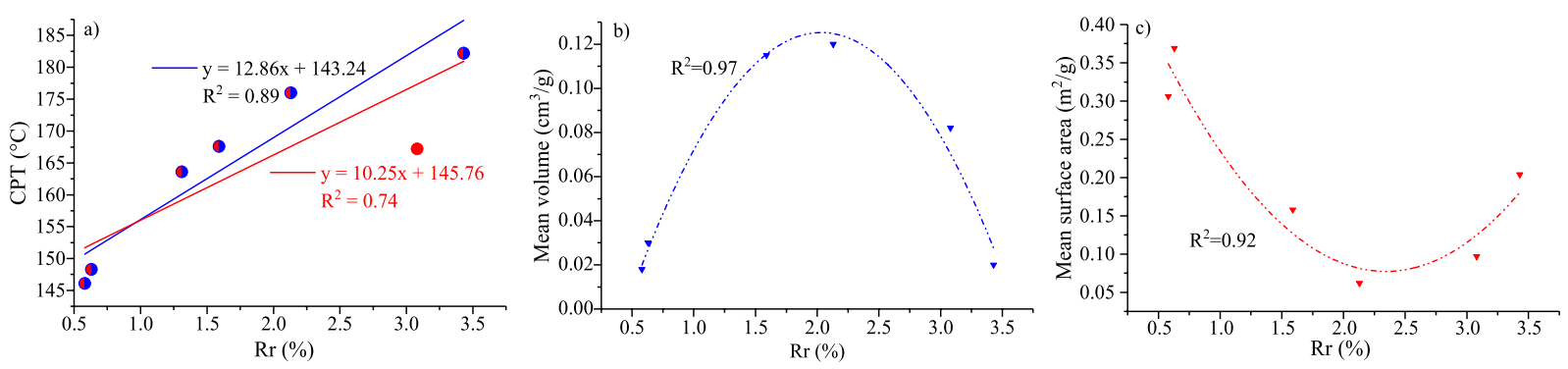

Figure 8. (a) Relationship between the vitrinite random reflectance $(\mathrm{Rr}, \%)$ and (a) the CPT, (b) mean volume $\left(\mathrm{cm}^{3} / \mathrm{g}\right)$, and $(\mathbf{c})$ mean surface area $\left(\mathrm{m}^{2} / \mathrm{g}\right)$.
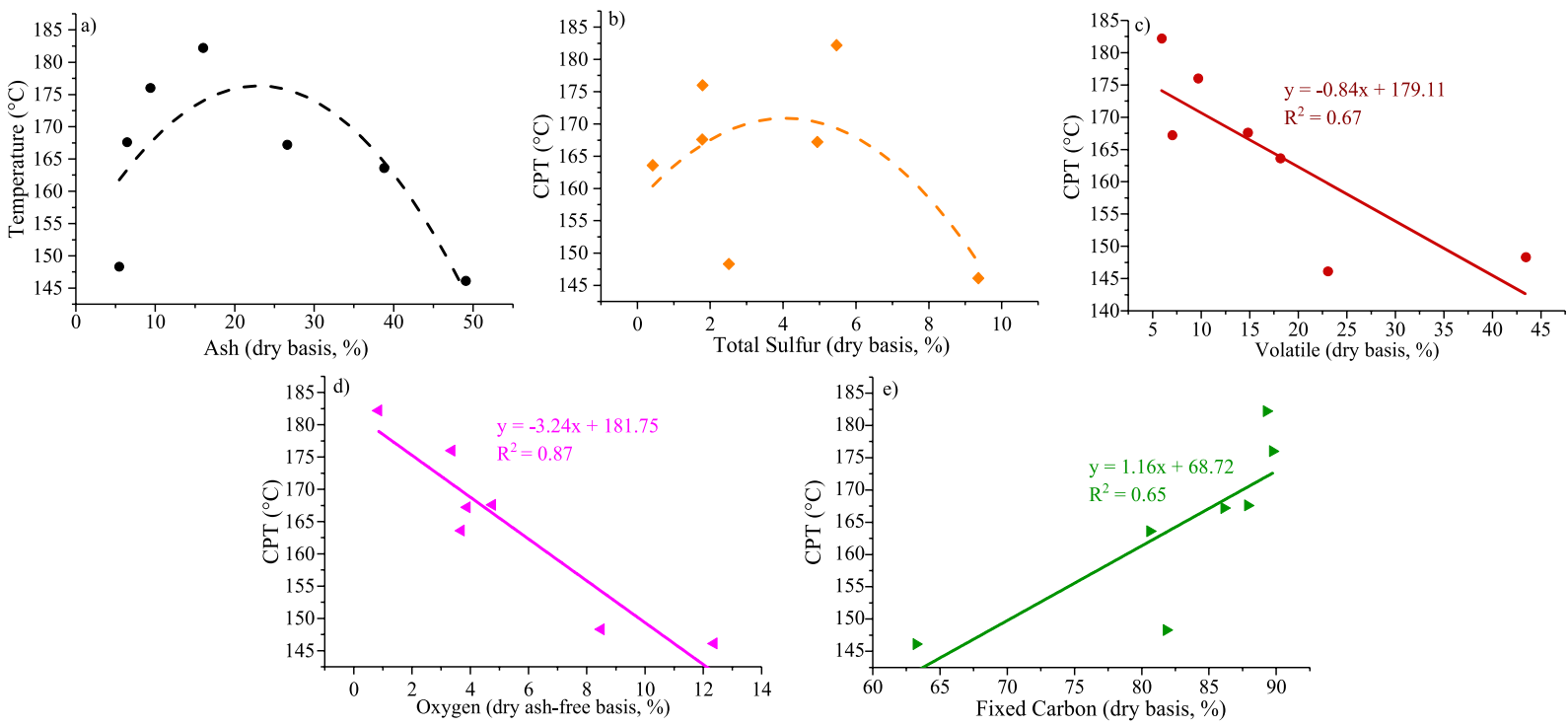

Figure 9. Relationship between the CPT and the (a) ash yield, (b) total sulfur content, (c) volatile matter, (d) oxygen content, and (e) fixed carbon content.

This may be owing to joint action factors such as maceral content, ash content, volatile matter, and tectonic deformations. The relationship between cumulative pore volume $V_{1}$ and $D_{1}$ is not clear, indicating that $V_{1}$ (adsorption-pores) may have little influence on the entropy dimension $\left(D_{1}\right)$ (Fig. 11b). The $D_{1}$ increases with increasing cumulative pore volume $V_{2}$ (Fig. 11c) and decreases with increasing cumulative pore volume $V_{3}$ (Fig. 11d), indicating that seepage-pores have an important influence on the entropy dimension $\left(D_{1}\right)$.

The Hurst exponent $(H)$ is used to quantify the degree of correlation on the logarithmic scale ${ }^{71}$. If $H>0.5$, the increments are correlated ${ }^{71}$. The Hurst exponent of all the samples exceeds more than $0.5(\mathrm{H}, 0.57-0.97)$, indicating the increments are correlated (Table 3). The Hurst exponents of samples $S_{1}$ and $S_{3}$ are 0.97 and 0.90 , respectively, which two data are close to the 1, reflecting the presence of strong persistence or positive autocorrelations ${ }^{49,71}$. The Hurst exponent has the same characteristics as entropy dimension (Fig. 12).

The width of $D(q)$ reflects the heterogeneity in the porosity distribution ${ }^{45,51}$. Sample $\mathrm{S}_{7}$ has the highest $D_{-10}$ $-D_{10}$ (the widest spectrum), indicating the highest heterogeneity over the entire pore size range among all the coal samples ${ }^{45,51}$. In contrast, sample $S_{1}$ has the lowest $D_{-10}-D_{10}$ (the narrowest spectrum), reflecting the lowest heterogeneity in the porosity distribution over the entire pore size range ${ }^{45,51}$. Samples $S_{2}$ and $S_{6}$ have wider right side $D(q)$ spectra than the left side $D(q)$ spectra, indicating high dominance of the high porosity concentrations ${ }^{45}$. However, samples $S_{1}, S_{4}, S_{5}$, and $S_{7}$ have wider left side $D(q)$ spectra than right side $D(q)$ spectra, indicating a small porosity concentration ${ }^{45}$. The widths of the $D(q)\left(D_{-10}-D_{10}, D_{0}-D_{10}\right.$, and $\left.D_{-10}-D_{0}\right)$ spectra increase with increasing vitrinite random reflectance $(R r, \%)$ (Fig. 13a). The relationship between cumulative pore volume $V_{1}$ and width of the $D(q)$ spectra is not clear, indicating that $V_{1}$ (adsorption-pores) may have little influence on the width of the $D(q)$ spectra (Fig. 13b). The width of the $D(q)$ spectra logarithmically decreases with increasing cumulative pore volume $V_{2}$ (Fig. 13c) and logarithmically increases with increasing cumulative pore volume $V_{3}$ (Fig. 13d). The above indicates that seepage-pores have an important influence on the heterogeneity in the porosity distribution.

Fractal dimensions and CPT. 1) Multiscale fractal dimensions and CPT. The relationships between the fractal dimensions of the Sierpinski model $\left(D_{s 1}\right.$ and $\left.D_{s 2}\right)$ and the CPT are shown in Fig. 11. The relationship between $D_{s 1}$ and the CPT can be divided into two distinct stages (Fig. 14a). In the first stage, the CPT first decreases, 

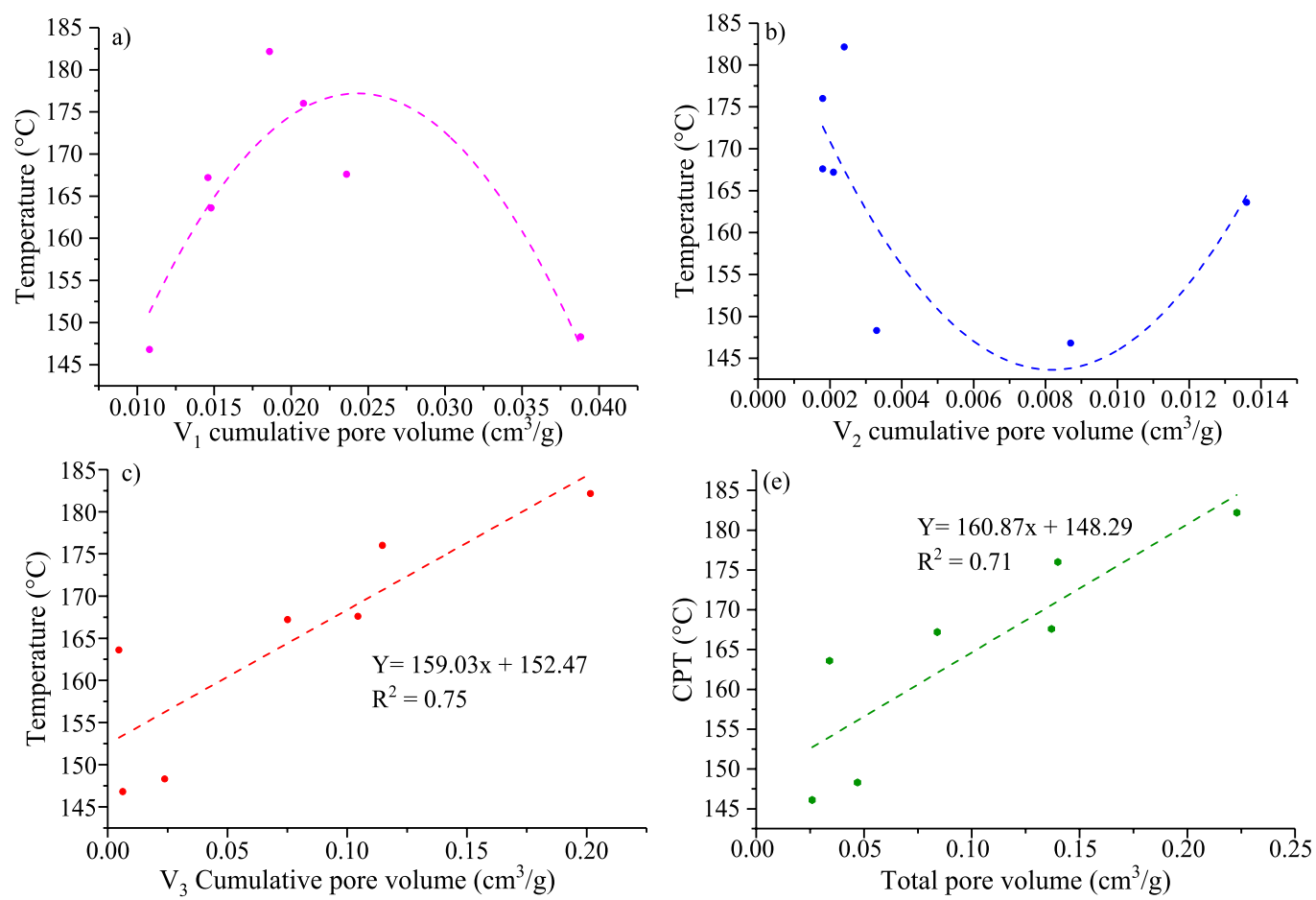

Figure 10. Relationship between CPT and the (a-d) pore volume distribution, and (e) total pore volume.
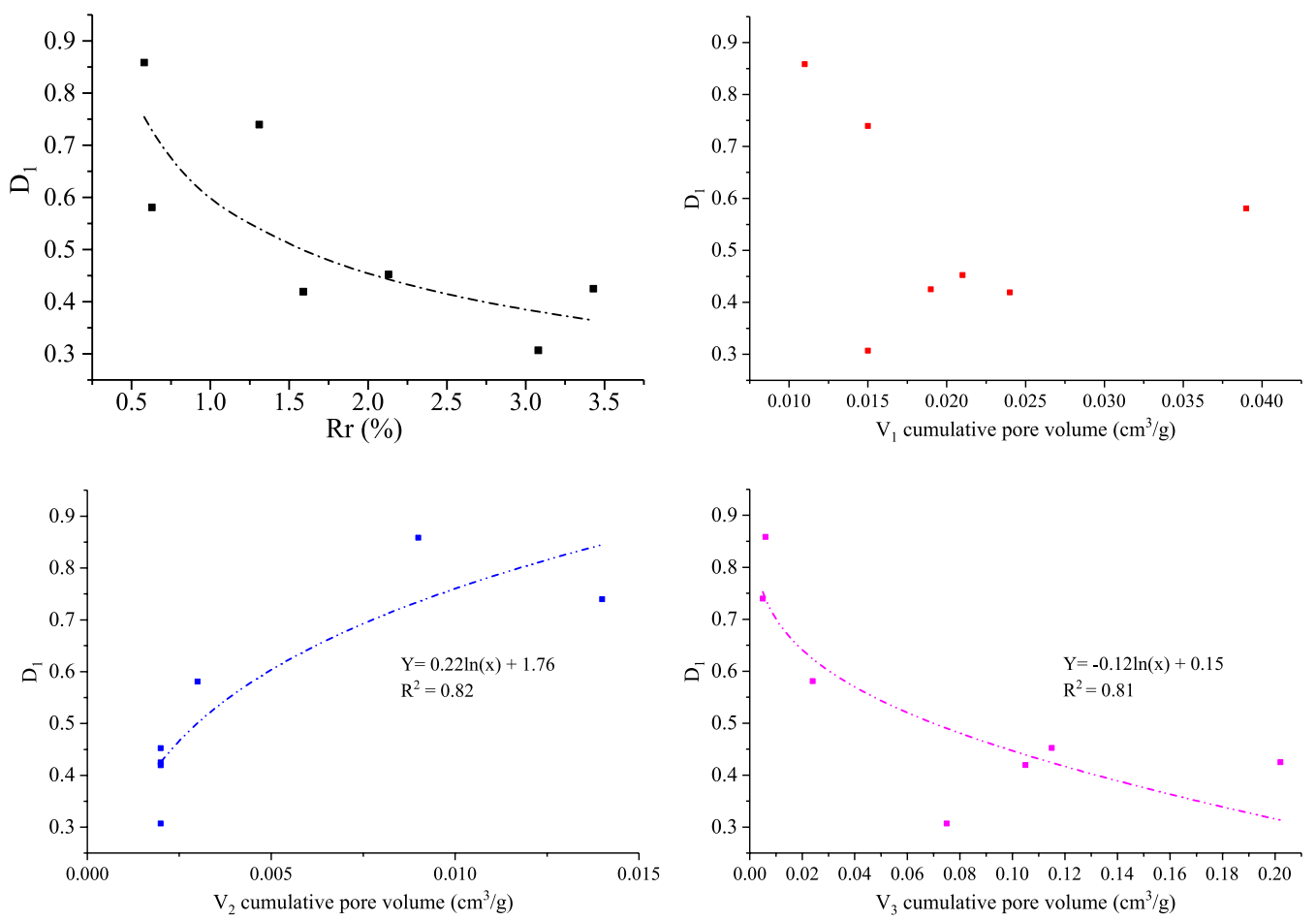

Figure 11. Relationship between $D_{1}$ and the (a) vitrinite random reflectance $(R r, \%)$ and (b-d) Cumulative pore volume $\left(V_{1}, V_{2}\right.$ and $\left.V_{3}\right)$.

and then increases with increasing $D_{s 1}$ (when $D_{s 1}>2.8$ ). With increasing $D_{s 2}$, the CPT decreases first (from 2.75 to 2.85), and then increases (Fig. 14b). Fractal dimensions $D_{g 1}$ of samples $S_{1}$ and $S_{3}$ are $>3$; however, most of the fractal dimensions, $D_{g 2}$, are $>3$, except of sample $S_{1}$. Fractal dimensions $D_{g 1}$ and $D_{g 2}$ are $>3$, which are not suitable to characterize the pore heterogeneity ${ }^{72,73}$. To prevent the interference of abnormal points, the data for fractal dimensions $>3$ were removed ${ }^{37}$. The relationship between $D_{g 1}$ and the CPT (without samples $\mathrm{S}_{1}$ and $\mathrm{S}_{2}$ ) 

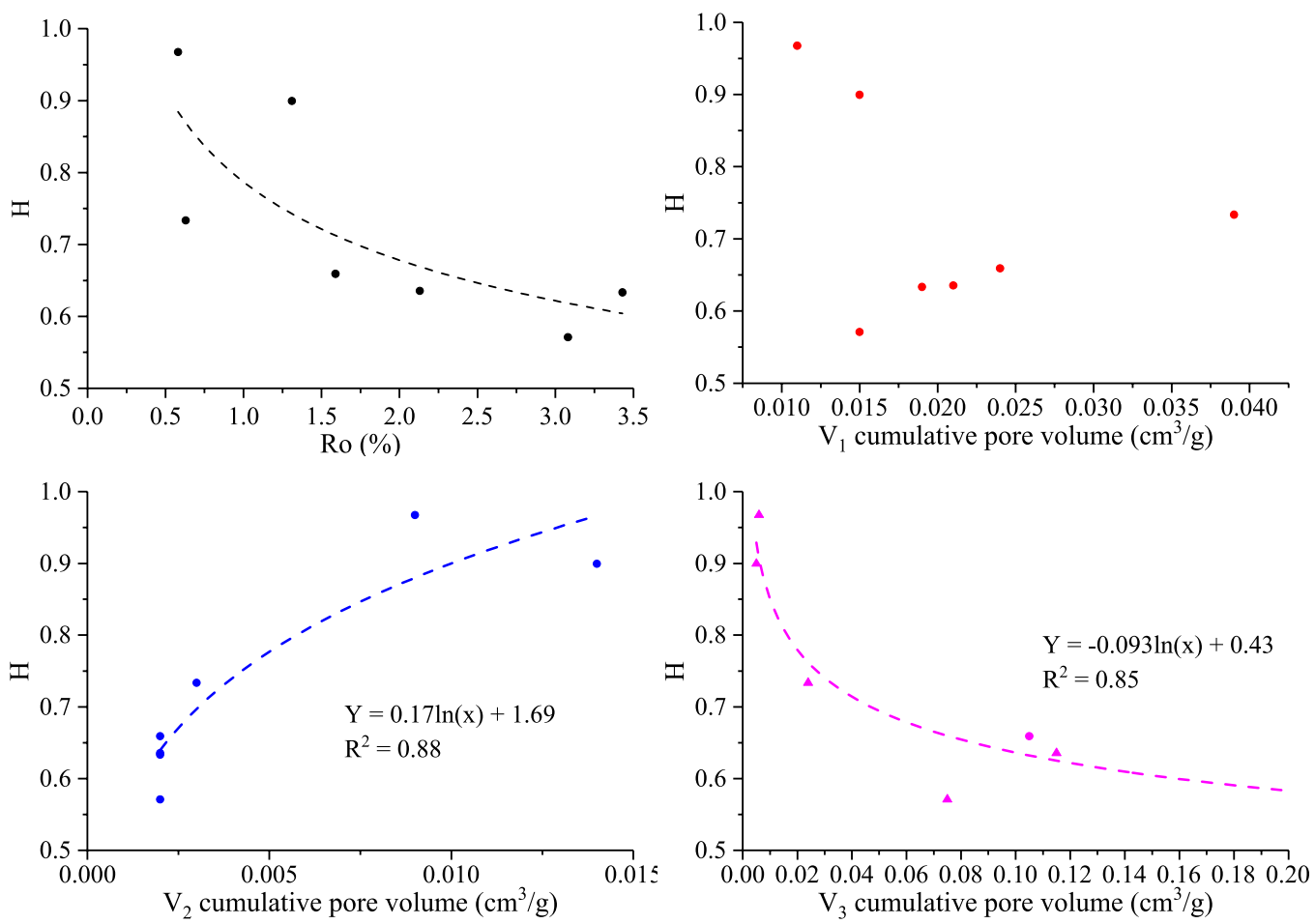

Figure 12. Relationship between the Hurst exponent $(H)$ and the (a) vitrinite random reflectance $(R r, \%)$ and (b-d) Cumulative pore volumes $\left(V_{1}, V_{2}\right.$, and $\left.V_{3}\right)$.
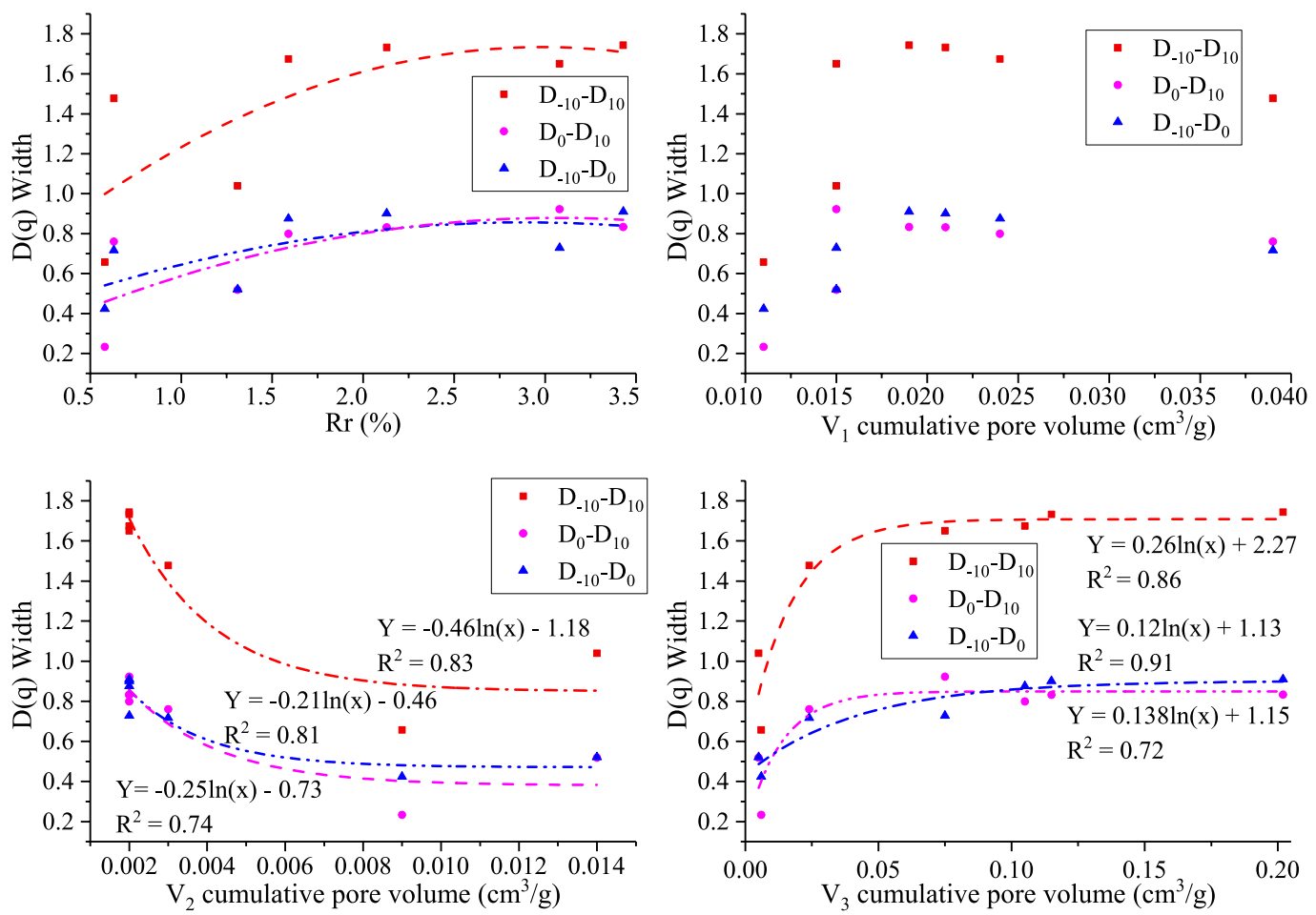

Figure 13. Width of the $\mathrm{D}(\mathrm{q})$ spectra $\left(D_{-10}-D_{10}, D_{0}-D_{10}\right.$, and $\left.D_{-10}-D_{0}\right)$ and the (a) vitrinite random reflectance $(\mathrm{Rr}, \%)$ and $(\mathbf{b}-\mathbf{d})$ cumulative pore volumes $\left(\mathrm{V}_{1}, \mathrm{~V}_{2}\right.$, and $\left.\mathrm{V}_{3}\right)$.

displays a similar trend as that between $D s$ and the CPT obtained from the Sierpinski model (Fig. 14c). When $D_{g 1}$ ranges from 2 to 2.6, the CPT decreased significantly. When $D_{g 1}$ becomes larger than 2.6, the CPT increases with increasing $D_{g 1}$. The above results demonstrate that the heterogeneities obtained from the Sierpinski and Sponge 

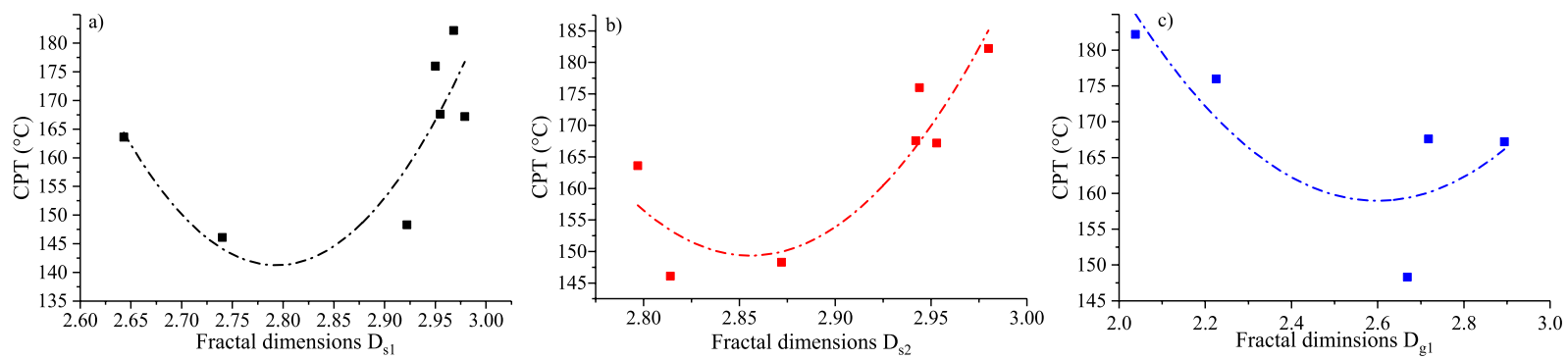

Figure 14. Relationship between the CPT and (a) fractal dimensions $D_{s 1}$, (b) fractal dimensions $D_{s 2}$, and (e) fractal dimensions $D_{g 1}$.
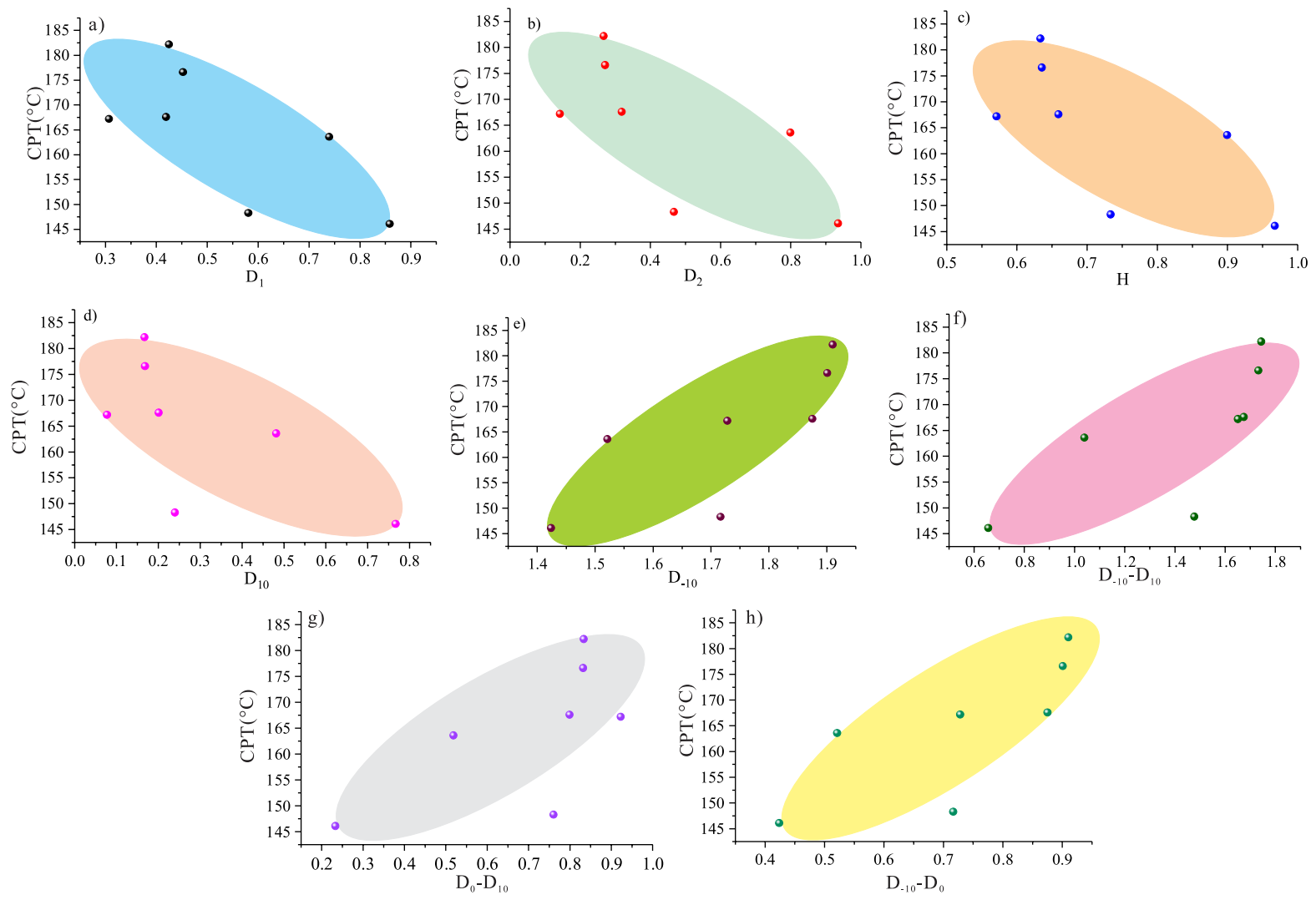

Figure 15. Relationship between the $\mathrm{CPT}$ and the multifractal parameters.

models do not present linear relationship with the tendency of coal to spontaneous combustion. However, a high heterogeneity (fractal dimensions $>2.8$ ) is associated with a low tendency of spontaneous combustion.

2) Multifractal fractal dimensions and CPT. Fig. 15 displays the correlation between the multifractal fractal dimensions $\left(D_{1}, D_{2}, H, D_{10}, D-_{10}, D_{-10}-D_{10}, D_{-10}-D_{0}, D_{0}-D_{10}\right)$ and the CPT. It can be found that the CPT decreases with increasing parameters $D_{1}, D_{2}, H$, and $D_{10}$, suggesting that a high degree of the distribution of the porosity quantifies the degree of correlation on the logarithmic scale. Further, the heterogeneity in the porosity distribution decreases the tendency of coal spontaneous combustion. The correlation between $D_{-10}, D_{-10}-D_{10}, D_{-10}-D_{0}$, and $D_{0}-D_{10}$ and the CPT is clear in that the CPT gradually increases with increasing $D_{-10}-D_{10}, D_{-10}-D_{0}$, and $D_{0}-D_{10}$. This indicates that the complexity of the local characterization pore structure decrease the spontaneous combustion propensity.

\section{Conclusions}

The conventional analysis and CPT measurements were conducted to obtain the properties of coal petrology and spontaneous combustion. Coal rank, volatile matter, oxygen content, and fixed carbon content were found to play important roles in spontaneous combustion.

The pore structure properties obtained from HPMI provided a direct measurement of coal physical properties. The cumulative pore volume of $V_{3}(>10000 \mathrm{~nm})$ and total pore volume have positive correlation with CPT. 
Multiscale and multifractal analyses were conducted to evaluate the pore size distribution. From the multiscale analysis, the relationship between the fractal dimensions $\left(D_{\mathrm{s} 1}, \mathrm{D}_{\mathrm{s} 2}\right.$, and $\left.\mathrm{D}_{\mathrm{g} 1}\right)$ and the CPT basically displayed a "U-shaped" tendency, with the minimum occurring at 2.6-2.85. Based on the multifractal analysis, a high degree of porosity distribution, quantified the degree of correlation on the logarithmic scale. Furthermore, the heterogeneity in the porosity distribution decreased the tendency of coal spontaneous combustion; therefore, a more complex local characterization pore structure lowered the spontaneous combustion propensity.

Received: 17 July 2019; Accepted: 24 January 2020;

Published online: 28 April 2020

\section{References}

1. Parsa, M. R., Tsukasaki, Y., Perkins, E. L. \& Chaffee, A. L. The effect of densification on brown coal physical properties and its spontaneous combustion propensity. Fuel 193, 54-64 (2017).

2. Fei, Y. et al. The spontaneous combustion behavior of some low rank coals and a range of dried products. Fuel 88, 1650-1655 (2009).

3. Song, Z. \& Kuenzer, C. Coal fires in China over the last decade: A comprehensive review. International Journal of Coal Geology 133, 72-99 (2014).

4. Gielisch H, K. C. Coal Fires a major Source of Greenhouse Gases-a forgotten problem. Environmental Risk Assessment and Remediation 2, 5-8 (2018).

5. Stracher, G. B. Coal fires burning around the world: A global catastrophe. International Journal of Coal Geology 59, 1-6 (2004).

6. Engle, M. A. et al. Gas emissions, minerals, and tars associated with three coal fires, Powder River Basin, USA. Sci Total Environ 420, 146-159 (2012).

7. Kuenzer, C. \& Stracher, G. B. Geomorphology of coal seam fires. Geomorphology 138, 209-222 (2012).

8. Kuenzer, C. et al. Uncontrolled coal fires and their environmental impacts: Investigating two arid mining regions in north-central China. Applied Geography 27, 42-62 (2007).

9. Finkelman, R. B. Potential health impacts of burning coal beds and waste banks. International Journal of Coal Geology 59, 19-24 (2004).

10. Deng, J. et al. Determination and prediction on "three zones" of coal spontaneous combustion in a gob of fully mechanized caving face. Fuel 211, 458-470 (2018).

11. Wang, D., Qi, X., Zhong, X. \& Gu, J. Test method for the propensity of coal to spontaneous combustion. Procedia Earth and Planetary Science 1, 20-26 (2009).

12. Küçük, A., Kadıoğlu, Y. \& Gülaboğlu, M. A study of spontaneous combustion characteristics of a Turkish lignite: particle size, moisture of coal, humidity of air. Combustion and Flame 133, 255-261 (2003).

13. Nimaje, D. S., Tripathy, D. P. \& Nanda, S. K. Development of regression models for assessing fire risk of some Indian coals. International Journal of Intelligent Systems and Applications 5, 52-58 (2013).

14. D. P. Tripathy, B. K. Pal, Spontaneous heating susceptibility of coals - evaluation based on experimental techniques. Journal of Mines, Metals and Fuels, Medium: X; Size: page(s) 236-243 (2001).

15. Cao, D., Fan, X., Shi, X., Wu, C. \& Wei, Y. Analysis of spontaneous combustion internal factors and division of spontaneous combustion types of coal seam in Wuda coalfield, Inner Mongolia. Journal of China Coal Society 30, 288-292 (2005).

16. B. Beamish, A. Arisoy, In Proceedings of the 12th US/North American Mine Ventilation Symposium., pp. 149-153 (2008).

17. Bhat, S. \& Agarwal, P. K. The effect of moisture condensation on the spontaneous combustibility of coal. Fuel 75, 1523-1532 (1996).

18. Kadioğlu, Y. \& Varamaz, M. The effect of moisture content and air-drying on spontaneous combustion characteristics of two Turkish lignitesa. Fuel 82, 1685-1693 (2003)

19. Su, H., Zhou, F., Li, J. \& Qi, H. Effects of oxygen supply on low-temperature oxidation of coal: A case study of Jurassic coal in Yima, China. Fuel 202, 446-454 (2017).

20. Sujanti, W. \& Zhang, D. K. A laboratory study of spontaneous combustion of coal: the influence of inorganic matter and reactor size. Fuel 78, 549-556 (1999).

21. Xuyao, Q., Wang, D., Milke, J. A. \& Zhong, X. Crossing point temperature of coal. Mining Science and Technology (China) 21, 255-260 (2011).

22. Nimaje, D. S. \& Tripathy, D. P. Characterization of some Indian coals to assess their liability to spontaneous combustion. Fuel 163, 139-147 (2016).

23. Chandra, D. \& Prasad, Y. V. S. Effect of Coalification on Spontaneous Combustion of Coals. International Journal of Coal Geology 16, 225-229 (1990).

24. Pattanaik, D. S., Behera, P. \& Singh, B. Spontaneous Combustibility Characterisation of the Chirimiri Coals, Koriya District, Chhatisgarh, India. International Journal of Geosciences 2, 336-347 (2011).

25. Clemens, A. H. \& Matheson, T. W. The role of moisture in the self-heating of low-rank coals. Fuel 75, 891-895 (1996)

26. Y. Zhang, X. Zhang, C. Liu, X. Yu, S. Hu, Impact of the crystallite parameters and coal ranks on oxidation and combustion properties of Carboniferous coals and Jurassic coals. Arabian Journal of Geosciences 11 (2018).

27. В. В. Ходот, Outburst of Coal and Coalbed Gas (Chinese Translation). (China Industry Press, Beijing, 1961).

28. Yao, Y., Liu, D., Tang, D., Tang, S. \& Huang, W. Fractal characterization of adsorption-pores of coals from North China: An investigation on $\mathrm{CH}_{4}$ adsorption capacity of coals. International Journal of Coal Geology 73, 27-42 (2008).

29. Kaji, F., Hishinuma, Y. \& Nakamura, Y. Low temperature oxidation of coals: effects of pore structure and coal composition. Fuel 64, 297-302 (1985).

30. Arisoy, A. \& Beamish, B. Reaction kinetics of coal oxidation at low temperatures. Fuel 159, 412-417 (2015).

31. Shi, Q. et al. Effects of igneous intrusions on the structure and spontaneous combustion propensity of coal: A case study of bituminous coal in Daxing Mine, China. Fuel 216, 181-189 (2018).

32. Carras, J. N. \& Young, B. C. Self-heating of coal and related materials: Models, application and test methods. Progress in Energy and Combustion Science 20, 1-15 (1994).

33. Wang, H., Dlugogorski, B. Z. \& Kennedy, E. M. Experimental Study on Low-Temperature Oxidation of an Australian Coal. Energy \& Fuels 13, 1173-1179 (1999).

34. Yang, M. \& Liu, W. M. The d-p band-inversion topological insulator in bismuth-based skutterudites. Sci Rep 4, 5131 (2014).

35. Karsner, G. G. \& Perlmutter, D. D. Reaction regimes in coal oxidation. AIChE Journal 27, 920-927 (1981).

36. Yu, S., Bo, J. \& Jiegang, L. Nanopore structural characteristics and their impact on methane adsorption and diffusion in low to medium tectonically deformed coals: Case study in the Huaibei coal field. Energy \& Fuels 31, 6711-6723 (2017).

37. Zhou, S. et al. Multi-scale fractal characterizations of lignite, subbituminous and high-volatile bituminous coals pores by mercury intrusion porosimetry. Journal of Natural Gas Science and Engineering 44, 338-350 (2017).

38. Zhao, J. et al. Coal seam porosity and fracture heterogeneity of macrolithotypes in the Hancheng Block, eastern margin, Ordos Basin, China. International Journal of Coal Geology 159, 18-29 (2016).

39. Liu, X. \& Nie, B. Fractal characteristics of coal samples utilizing image analysis and gas adsorption. Fuel 182, 314-322 (2016). 
40. Zhang, J. et al. Characterization of Full-Sized Pore Structure and Fractal Characteristics of Marine-Continental Transitional Longtan Formation Shale of Sichuan Basin, South China. Energy \& Fuels 31, 10490-10504 (2017).

41. Liu, Y., Zhu, Y., Li, W., Zhang, C. \& Wang, Y. Ultra micropores in macromolecular structure of subbituminous coal vitrinite. Fuel 210, 298-306 (2017).

42. Liu, Y. et al. Molecular simulation of methane adsorption in shale based on grand canonical Monte Carlo method and pore size distribution. Journal of Natural Gas Science and Engineering 30, 119-126 (2016).

43. Schlueter, E. M., Zimmerman, R. W., Witherspoon, P. A. \& Cook, N. G. W. The fractal dimension of pores in sedimentary rocks and its influence on permeability. Engineering Geology 48, 199-215 (1997).

44. Cai, Y., liu, D., Yao, Y., Li, J. \& Liu, J. Fractal characteristics of coal pores based on classic geometry and thermodynamics models. Acta Geologica Sinica 85, 1150-1162 (2011).

45. Yu, S., Bo, J., Pei, S. \& Jiahao, W. Matrix compression and multifractal characterization for tectonically deformed coals by $\mathrm{Hg}$ porosimetry. Fuel 211, 661-675 (2018).

46. Yu, S., Bo, J., Fengli, L. \& Jiegang, L. Structure and fractal characteristic of micro- and meso-pores in low, middle-rank tectonic deformed coals by CO 2 and N 2 adsorption. Microporous and Mesoporous Materials 253, 191-202 (2017).

47. Dathe, A., Tarquis, A. M. \& Perrier, E. Multifractal analysis of the pore- and solid-phases in binary two-dimensional images of natural porous structures. Geoderma 134, 318-326 (2006).

48. Vázquez, E. V., Ferreiro, J. P., Miranda, J. G. V. \& González, A. P. Multifractal Analysis of Pore Size Distributions as Affected by Simulated Rainfall. Vadose Zone Journal 7, 500 (2008).

49. San José Martínez, F. et al. Multifractal analysis of discretized X-ray CT images for the characterization of soil macropore structures. Geoderma 156, 32-42 (2010).

50. De, W., Fu, B., Zhao, W., Hu, H. \& Wang, Y. Multifractal characteristics of soil particle size distribution under different land-use types on the Loess Plateau, China. Catena 72, 29-36 (2008).

51. Li, W., Liu, H. \& Song, X. Multifractal analysis of $\mathrm{Hg}$ pore size distributions of tectonically deformed coals. International Journal of Coal Geology 144-145, 138-152 (2015).

52. Wang, D., Dou, G., Zhong, X., Xin, H. \& Qin, B. An experimental approach to selecting chemical inhibitors to retard the spontaneous combustion of coal. Fuel 117, 218-223 (2014).

53. B. B. Mandelbrot, The fractal geometry of nature /Revised and enlarged edition. New York W.h.freeman \& Co.p -1, (1983).

54. International Standard, in ISO Standards, Geneva. (2005), vol. 11760.

55. Zhang, S., Tang, S., Tang, D., Huang, W. \& Pan, Z. Determining fractal dimensions of coal pores by FHH model: Problems and effects. Journal of Natural Gas Science and Engineering 21, 929-939 (2014).

56. Muller, J. Characterization of pore space in chalk by multifractal analysis. Journal of Hydrology 187, 215-222 (1999).

57. Paz-Ferreiro, J., Vázquez, E. V. \& Miranda, J. G. V. Assessing soil particle-size distribution on experimental plots with similar texture under different management systems using multifractal parameters. Geoderma 160, 47-56 (2010).

58. Khatami, R. \& Levendis, Y. A. An overview of coal rank influence on ignition and combustion phenomena at the particle level. Combustion and Flame 164, 22-34 (2016).

59. Beamish, B. B., Barakat, M. A. \& St George, J. D. Spontaneous-combustion propensity of New Zealand coals under adiabatic conditions. International Journal of Coal Geology 45, 217-224 (2001).

60. Zhou, F. B. \& Wang, D. M. Directory of recent testing methods for the propensity of coal to spontaneous combustion. Journal of Fire Sciences 22, 91-96 (2004)

61. Avila, C., Wu, T. \& Lester, E. Petrographic characterization of coals as a tool to detect spontaneous combustion potential. Fuel 125, 173-182 (2014).

62. Liu, Y. et al. Molecular structure controls on micropore evolution in coal vitrinite during coalification. International Journal of Coal Geology 199, 19-30 (2018).

63. RM, B. G. Y, Abrupt changes (jumps) in reflectance values and chemical compositions of artificial charcoals and inertinite in coals. International Journal of Coal Geology 38, 237-260 (1999).

64. Li, W., Zhu, Y., Wang, G. \& Jiang, B. Characterization of coalification jumps during high rank coal chemical structure evolution. Fuel 185, 298-304 (2016).

65. KAYMAKÇI, E. \& Didari, V. Relations between Coal Properties and Spontaneous Combustion Parameters. Turkish Journal of Engineering and Environmental Sciences 26, 59-64 (2002).

66. Shi, Q., Qin, B., Bi, Q. \& Qu, B. An experimental study on the effect of igneous intrusions on chemical structure and combustion characteristics of coal in Daxing Mine, China. Fuel 226, 307-315 (2018).

67. Ge, L., Zhang, Y., Wang, Z., Zhou, J. \& Cen, K. Effects of microwave irradiation treatment on physicochemical characteristics of Chinese low-rank coals. Energy Conversion and Management 71, 84-91 (2013).

68. Ogunsola, O. I. \& Mikula, R. J. A study of spontaneous combustion characteristics of Nigerian coals. Fuel 70, 258-261 (1991).

69. Muthuraman, M., Namioka, T. \& Yoshikawa, K. Characteristics of co-combustion and kinetic study on hydrothermally treated municipal solid waste with different rank coals: A thermogravimetric analysis. Applied Energy 87, 141-148 (2010).

70. Caniego, F. J., Martí, M. A. \& José, F. S. Rényi dimensions of soil pore size distribution. Geoderma 112, 205-216 (2003).

71. Norouzzadeh, P., Dullaert, W. \& Rahmani, B. Anti-correlation and multifractal features of Spain electricity spot market. Physica A: Statistical Mechanics and its Applications 380, 333-342 (2007).

72. Mahamud, M., López, Ó., Pis, J. J. \& Pajares, J. A. Textural characterization of coals using fractal analysis. Fuel Processing Technology 81, 127-142 (2003).

73. Friesen, W. I. \& Ogunsolaf, O. I. Mercury porosimetry of upgraded western Canadian coals. Fuel 74, 604-609 (1995).

\section{Acknowledgements}

This work was supported by National Natural Science Foudation of China (Nos. 41430640 and 41672153 ) and the Scientific Research Foundation of Key Laboratory of Coalbed Methane Resource \& Reservoir Formation Process, Ministry of Education (No. 2017-002).

\section{Author contributions}

Zhang Yu: Data processing and paper writing. Zhang Xueqing: Samples preparation. Yang Wen: Samples collection. Xin Haihui: Experimental test. Hu Sherong: Funding and ideas. Song Yu: Method and paper revision.

\section{Competing interests}

The authors declare no competing interests.

\section{Additional information}

Correspondence and requests for materials should be addressed to H.S. or S.Y. 
Reprints and permissions information is available at www.nature.com/reprints.

Publisher's note Springer Nature remains neutral with regard to jurisdictional claims in published maps and institutional affiliations.

(c) (i) Open Access This article is licensed under a Creative Commons Attribution 4.0 International License, which permits use, sharing, adaptation, distribution and reproduction in any medium or format, as long as you give appropriate credit to the original author(s) and the source, provide a link to the Creative Commons license, and indicate if changes were made. The images or other third party material in this article are included in the article's Creative Commons license, unless indicated otherwise in a credit line to the material. If material is not included in the article's Creative Commons license and your intended use is not permitted by statutory regulation or exceeds the permitted use, you will need to obtain permission directly from the copyright holder. To view a copy of this license, visit http://creativecommons.org/licenses/by/4.0/.

(C) The Author(s) 2020 